\title{
Enhanced Time-Domain Detection of the Activation Frequency of Atrial Fibrillation
}

\author{
Ráhel Zölei-Szénási*, Gergely Vadai*, Róbert Pap ${ }^{* *}$, Zoltán Gingl* \\ * University of Szeged, Department of Technical Informatics, Szeged, Hungary \\ ** University of Szeged, Second Department of Medicine and Cardiology Centre, Szeged, Hungary \\ szenasi.rahel@stud.u-szeged.hu,vadaig@inf.u-szeged.hu,pap.paprobert@gmail.com, gingl@inf.u-szeged.hu
}

\begin{abstract}
Atrial fibrillation is the most common sustained cardiac arrhythmia. To determine the main frequency of the atrial fibrillation the frequency-domain analysis is used mostly, however it is unreliable in several cases. This insufficiency led to the demand of using time-domain analysis. Although these attempts seemed to be promising, there have remained several limitations. In our research we developed an enhanced algorithm for time-domain analysis. We defined two indicators which characterized the signals and the reliability of the method well. As compared to the frequency-domain analysis our algorithm can widen the applicability of time-domain analysis since in several cases when the results of the frequency-domain analysis are unreliable - it can be applied still reliably. As a complementation of the frequency-domain analysis our algorithm can be an extremely helpful tool in medical practice.
\end{abstract}

\section{INTRODUCTION}

Atrial fibrillation is the most common sustained cardiac arrhythmia. Nowadays, ca. $45 \%$ of the hospital admissions related to cardiac arrhythmia is caused by atrial fibrillation [1]. By 2050 the number of involved patients will be doubled in developed countries [2, 3]. In medical practice spectral-domain analysis of the ECG signal is used to determine the dominant frequency which is used to characterize the activation frequency of atrial fibrillation. Dominant frequency as a concept means the highest peak in the spectrum of the ECG signal however, it can be biased especially for signals with low regularity index or double-potentials, since the frequency of the highest peak does not belong to the activation frequency of the atrial fibrillation. This insufficiency led to the demand of using time-domain analysis.

Although several studies have already discussed this issue, there have remained several limitations of the applied time-domain methods. Mostly the investigations were restricted to signals with high signal-to-noise ratio and a multiple-stage conditioning was applied before the identification of the peaks [4-7].

The purpose of this study was to develop an algorithm for time-domain analysis which gives reliable results for signals with lower regularity by finding appropriate indicators. Furthermore, it was also desirable to achieve this by less signal-conditioning. To evaluate our algorithm, firstly we compared its frequency results to the outcomes of the frequency-domain analysis with high regularity index. Secondly, we investigated the changes of the frequencies obtained by the two techniques for signals with low regularity index. Finally, we defined a new

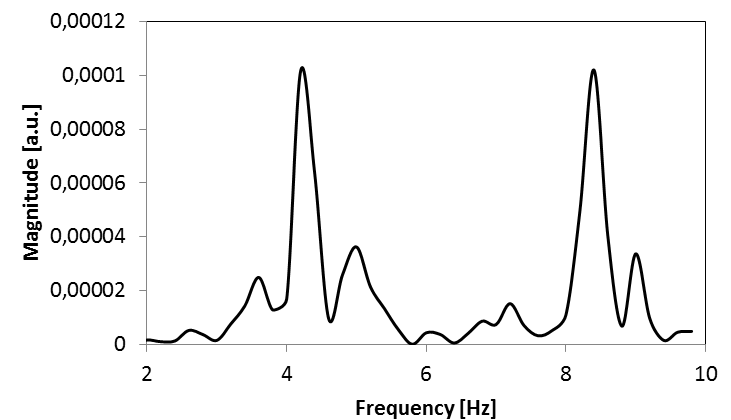

Figure 1. An example for the spectrum of an ECG signal with double-potentials

regularity index for time-domain analysis to demonstrate its reliability compared to frequency-domain analysis.

\section{METHODS}

In our research we used signals from 28 patients which were recorded at the Second Department of Internal Medicine and Cardiology Centre at University of Szeged. From our investigation we excluded those signals which were recorded on the skin-surface. Further conditions and more detailed description of the recordings can be found in [8].

As in the standard protocol, first we applied a third order Butterworth filter as a band-pass filter with a cutoff $40 / 250 \mathrm{~Hz}$ [9]. After that, unlike the common practice, we did not make a reduction, rectification or further signal conditioning but we calculated the derivate of our signal numerically by using second order central method. We searched for the plus-minus transitions which gave the places of the peaks in the original signal. This parameter has a similar meaning to the peak-threshold therefore we used the absolute value of the transition as the main parameter of the algorithm. To avoid multiple-detection of a single beat, we introduced a similar restriction like Seungyup Lee at el. in [4], so from the points detected in $60 \mathrm{~ms}$, we calculated a mean value which represented the given peak. In this way we could reach a beat-to-beat detection and determination of frequency from cycle length. On Fig. 2 can be seen two signals with different amplitudes with different absolute value of transition.

To find an appropriate indicator, we tested our algorithm for signals which had high regularity index for the frequency-domain, since in these cases the results of the frequency-domain analysis were reliable. We 

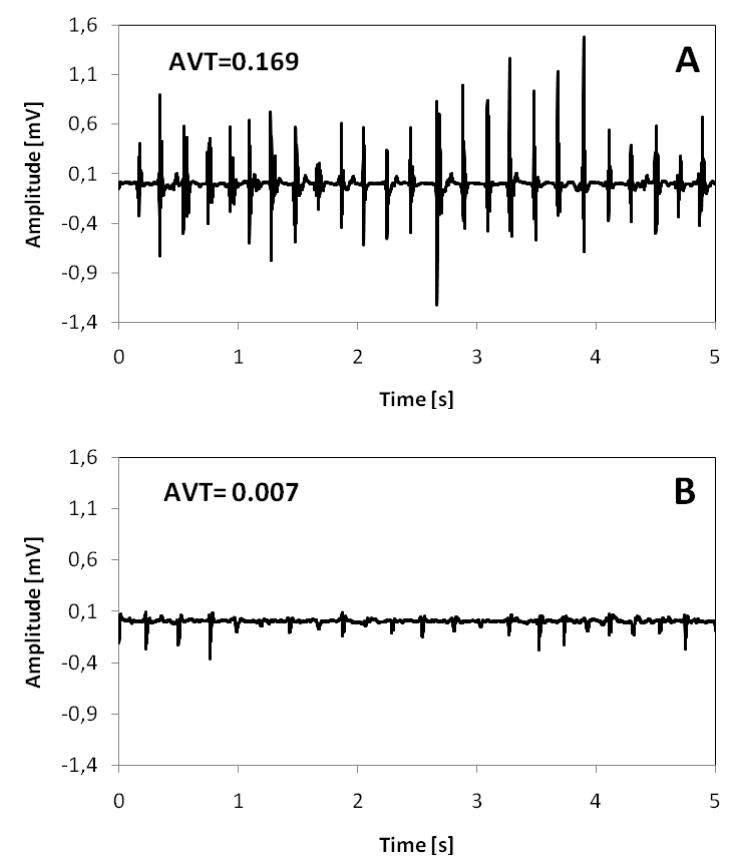

Figure 2. Two ECG signals with different amplitudes. For them, the optimal absolute value of transitions (AVT) differ greatly

compared the resulting dominant frequency of the frequency-domain analysis to the mean and mode of the determined frequencies of the time-domain analysis. We found that the mode is in great correspondence with dominant frequency, as it can be seen later in the Results and Discussion part. Although our algorithm was adaptive by the changeable absolute value of transition, we wanted to automate the process, therefore we complemented the algorithm. The main idea of the optimization was the standard deviation of the modes. For several given absolute value of transitions, we rolled a 5-second-long window through a 60-second-long signal and we calculated modes and their standard deviation. The algorithm selected the absolute value of transition which the smallest standard deviation belonged to.

Time-domain analysis is a very useful tool since it is not necessary to declare a biologically reasonable maximum frequency as we should in frequency-domain analysis. Moreover, it is less sensible for the examined signal length.

\section{RESULTS AND DISCUSSION}

\section{A. Mode is a better indicator than mean in time-domain} analysis.

According to the literature, mean is used to determine the activation frequency of the atrial fibrillation. In the case of high signal-to-noise ratio or well-filtered signals it can be reliable, however in other cases it can be misleading since the spilling points can garble the result, as we can see on Fig. 3. With choosing mode to the appropriate indicator we can reduce the effect of spilling points which could be caused by false detection or shortterm changes in frequency.
B. The resulted frequencies of the time-domain analysis are less sensible for the examined signal length.

In frequency-domain regularity index means the ratio of the area under dominant frequency and the whole spectral range which is examined. When the regularity index was lower than 0.4 (it could change between 0 and 1) it was not clear whether the dominant frequency and the activation frequency was the same, since the dominant frequency changed by the time. Sometimes the dominant frequency alternated between the fundamental and its overtone frequency - similarly to double-potential problem - however, it was not uncommon to change randomly.

Therefore we calculated the trend-line of dominant frequency - from frequency-domain - and mode - from time-domain - for 5, 10 and 15-second-long windows. As it can be seen on Fig. 3, the time-domain analysis is less
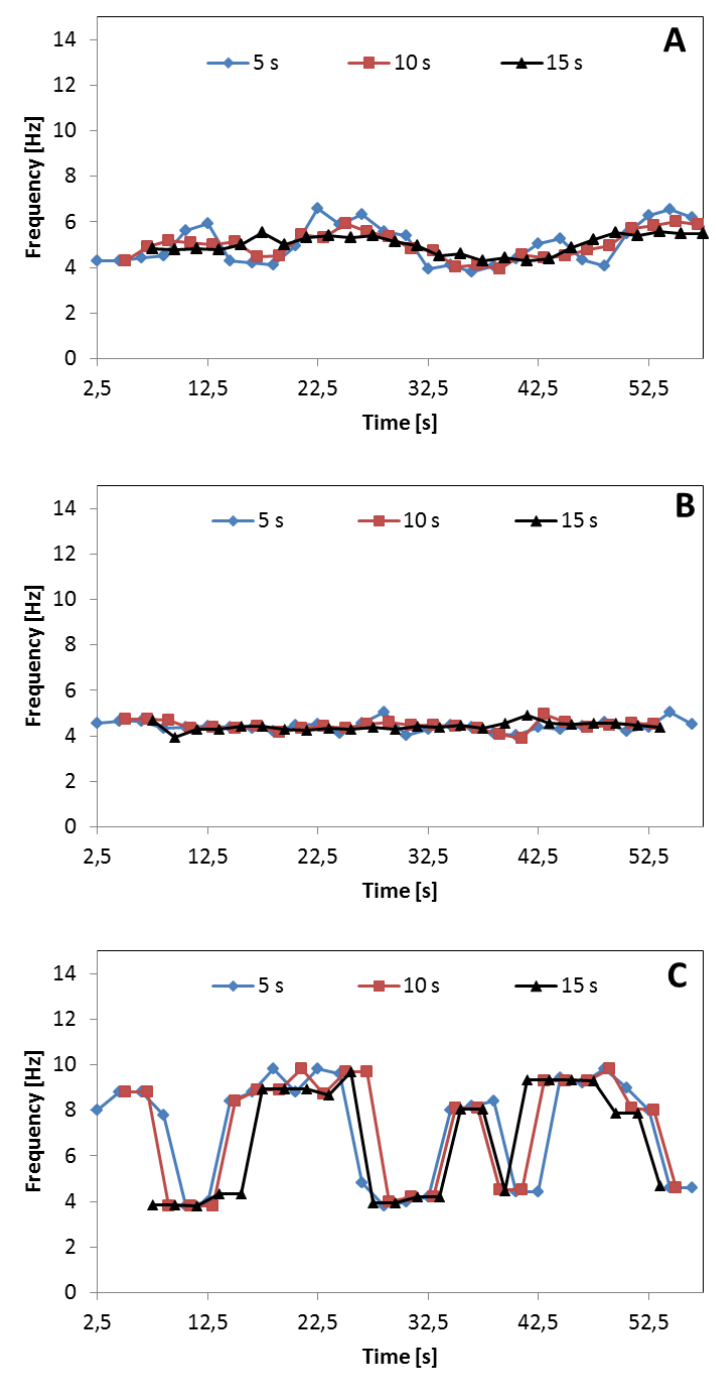

Figure 3. The main frequency of the atrial fibrillation determined by the use of three different methods with 5, 10, and 15 secondlong-window for the signal presented on Fig. 2B. On Fig. A the average of the inverse beat-to-beat cycle length within the applied window can be seen. On Fig. B the mode of the inverse beat-tobeat cycle length is plotted. On Fig. $\mathrm{C}$ the dominant frequency determined by the frequency-domain analysis can be seen. The advantage of the mode is clear as it automatically filters out spilling values moreover, it is much less sensible for the applied windowlength as compared to the frequency-domain analysis 
sensible for the examined signal length, and in the case of double-potentials, time-domain analysis could make clear which the activation frequency was. This kind of investigation gave a chance to test the stability of the main frequency in time as well.

C. For signals with high signal-to-noise ratio and high regularity index in frequency-domain, the timedomain analysis correspond well to the frequencydomain analysis. Furthermore time-domain analysis can be used reliably for signals with lower regularity index when spectrum-domain analysis is not definite.

As we mentioned previously, signals with high signalto-noise ratio and regularity index in frequency-domain $(0.6$ < regularity index $)$ have reliable results for the activation frequency, therefore we compared our results from the time-domain analysis - to them. According to Fig. 4, there was an obvious correspondence. Continuing the investigation we tried the algorithm on signals with lower regularity index. As a conclusion we could state that this kind of time-domain analysis widened the applicability, with this method it is possible to analyze those signals where frequency-domain analysis is unreliable however there is a reasonable limitation because of the signal-to-noise ratio.

D. To characterize the reliability of the resulting frequency from time-domain analysis we introduce a new regularity index for time-domain.

To test the reliability of our algorithm we wanted to define a similar index for our time-domain analysis like
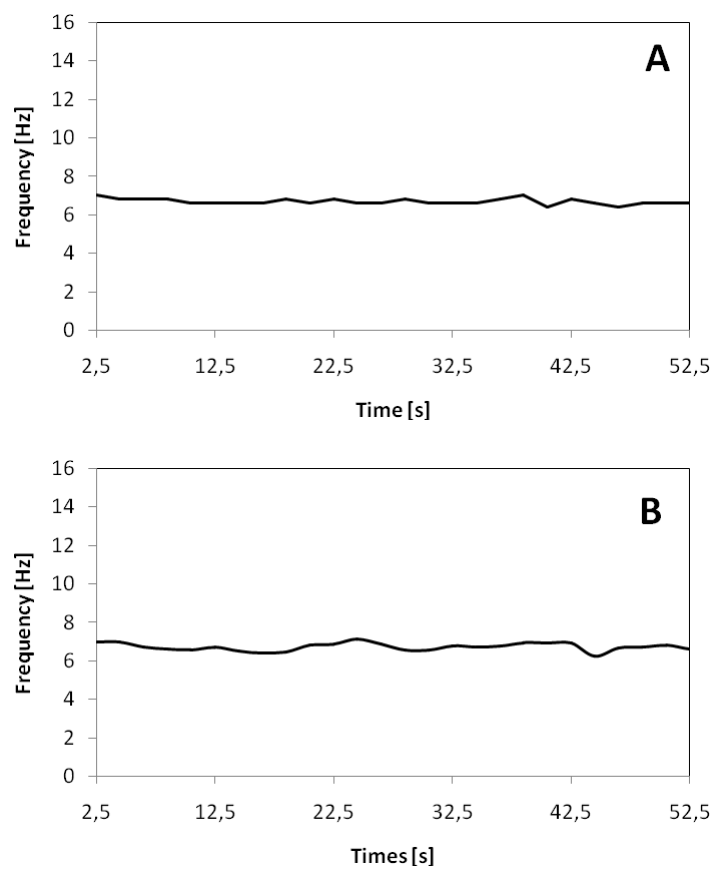

Figure 4. Validating the algorithm for a signal with high regularity index by using a 5 second-long window. On Fig. A the dominant frequency determined by the frequency-domain analysis is can be seen. On Fig. B the mode of the inverse beat-to-beat cycle length is plotted. There is a great correspondence between the commonly used and our enhanced method regularity index in frequency-domain. We counted the number of beats which had the value in the range of mode and plus-minus half of the standard deviation, then the number of beats in the given part of the signal. In this way regularity index for time-domain can be defined as the ratio of these numbers.
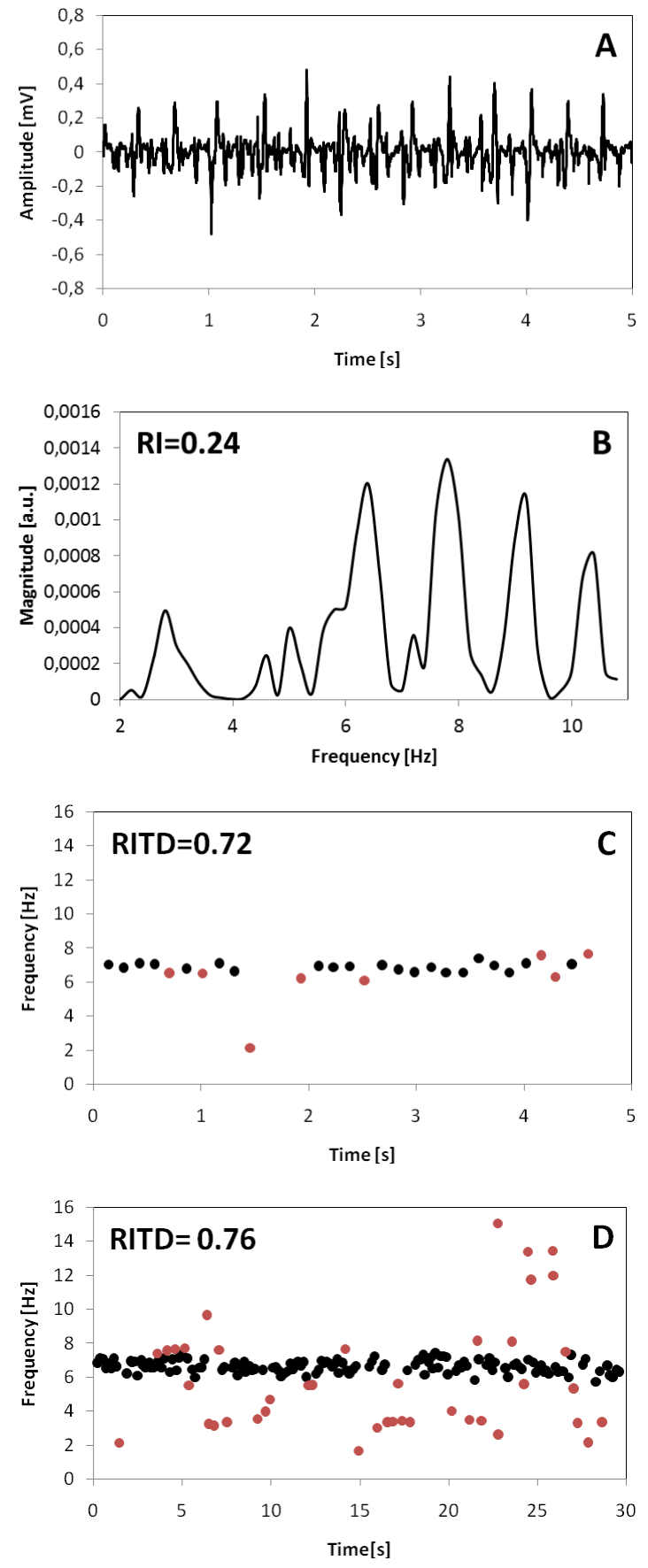

Figure 5. Illustration of the newly defined regularity index for time-domain analysis. On Fig. A the examined ECG signal can be seen. On Fig. B the Fourier spectrum (resolution $0.2 \mathrm{~Hz}$ ) is plotted On Fig. $\mathrm{C}$ the black points illustrate the range of mode and plusminus half of the standard deviation, and the red points show the spilling points for the first 5 seconds of the signal. On Fig. D the same can be seen - like Fig. C - for the first 30 seconds of the signal. The regularity indexes for the frequency-domain (RI) and the time-domain analysis (RITD) are also marked 


\section{CONCLUSION}

Nowadays determining the activation frequency of the atrial fibrillation is still a challenge. The frequencydomain analysis could not handle the case of doublepotentials and several others; therefore there were some attempts to develop time-domain analysis. These methods had a lot of limitations (e.g. high signal-to-noise ratio).

The time-domain analysis - which was developed by us - was tested and validated on signals with high and lower regularity index in frequency-domain for several windowlengths. The chosen indicator and the newly defined regularity index for time-domain served as reliable characteristics of the signals. It could help to identify the real activation frequencies in the case of double-potentials and widened the range of applicability. In sum, our timedomain analysis is accurate and can be a useful tool in the medical practice.

\section{ACKNOWLEDGMENT}

This research was realized in the frames of TÁMOP 4.2.4. A/2-11-1-2012-0001 „National Excellence Program - Elaborating and operating an inland student and researcher personal support system" The project was subsidized by the European Union and co-financed by the European Social Fund.

\section{REFERENCES}

[1] A. Mihálcz, Cs. Földesi, A. Kardos, K. Ladunga and T. SziliTörök, "The efficacy of class III D,L sotalol in treatment of left atrial tachycardia after pulmonary vein isolation," Orv. Hetil., vol. 150, no. 36, pp. 1694-1700, 2009.
[2] J. Tomcsányi, B. Bózsik, Gy. Rokszin, Zs. Abonyi-Tóth and L Katona, "The prevalence of atrial fibrillation in Hungary," Orv. Hetil., vol. 153, no. 9, pp. 339-342, 2012.

[3] A. Bajpai, I. Savelieva and AJ. Camm, "Epidemiology and Economic Burden of Atrial Fibrillation," US Cardiology, vol. 4, no. 1, pp. 14-17, 2007.

[4] S. Lee, K. Ryu, AL. Waldo, CM. Khrestian, DM. Durand and J. Sahadevan, "An algorithm to measure beat-to-beat cycle lengths for assessment of atrial electrogram rate and regularity during atrial fibrillation," J. Cardiovasc. Electrophysiol., vol. 24, no. 2, pp. 199-206, 2013.

[5] YJ. Lin, MT. Lo, C. Lin, SL. Chang, LW. Lo, YF. Hu, TF. Chao, CH Li, YC. Chang, WH. Hsieh, FP. Chung, HM. Tsao, HY. Chang, NE. Huang and SA. Chen, "Nonlinear analysis of fibrillatory electrogram similarity to optimize the detection of complex fractionated electrograms during persistent atrial fibrillation," J. Cardiovasc. Electrophysiol., vol. 24, no. 3, pp. 280-289, 2013.

[6] F. Ravelli, M. Masè, A. Cristoforetti, M. Del Greco, M. Centonze, M. Marini and M. Disertori, "Anatomic Localization of Rapid Repetitive Sources in Persistent Atrial Fibrillation: Fusion of Biatrial CT Images With Wave Similarity/Cycle Length Maps," JACC: Cardiovascular Imaging., vol. 5, no. 12, pp. 1211-1220, 2012.

[7] J. Ng, H. A. Kadish and J. J. Goldberger, "Effect of electrogram characteristics on the relationship of dominant frequency to atrial activation rate in atrial fibrillation," Heart Rhythm, vol. 3, no. 11, pp. 1295-1305, 2013.

[8] VB. Traykov, R. Pap, Z. Gingl, S. Chadaide, HM. Haqqani, G. Klausz, R. Gallardo, T. Forster, DJ. Callans and L. Sághy, "Role of triggering pulmonary veins in the maintenance of sustained paroxysmal atrial fibrillation," Pacing Clin. Electrophysiol., vol. 36, no. 7, pp. 845-854, 2013.

[9] J. Ng and JJ. Goldberger, "Understanding and interpreting dominant frequency analysis of AF electrograms," J. Cardiovasc. Electrophysiol., vol. 18, no. 6, pp. 680-685, 2007. 